\title{
Intestinal tuberculosis or Crohn's disease: a review of the diagnostic models designed to differentiate between these two gastrointestinal diseases
}

\author{
Julajak Limsrivilai, Nonthalee Pausawasdi \\ Division of Gastroenterology, Department of Medicine, Faculty of Medicine Siriraj Hospital, Mahidol University, Bangkok, Thailand
}

Differentiating Crohn's disease (CD) from intestinal tuberculosis (ITB) is a diagnostic dilemma, particularly in regions where ITB is prevalent and CD incidence is increasing, because both diseases can present quite similarly, and diagnostic tests to identify Mycobacterium tuberculosis in tissue samples have rather poor sensitivity. Studies that were conducted to determine the factors that differentiate CD from ITB identified some significant characteristics, but none of those characteristics are exclusive to either ITB or CD. Many diagnostic models or scoring systems that use one to several diagnostic parameters have been proposed to help distinguish these two intestinal diseases. Early models consisted of parameters common to routine clinical practice, such as clinical features, and endoscopic and pathologic findings. The later models also include more advanced diagnostic parameters like high-resolution imaging and serological testing. However, the number and types of parameters differ among diagnostic models, and the systems used to calculate scoring also vary from model to model. Enhanced awareness and understanding of the currently available diagnostic models will help physicians determine which model(s) is/are most suitable for differentiating CD from ITB in their clinical practice. (Intest Res 2021;19:21-32)

Key Words: Intestinal tuberculosis; Crohn disease; Diagnosis

\section{INTRODUCTION}

Differentiating intestinal tuberculosis (ITB) from Crohn's disease (CD) is important, particularly in regions where ITB and $\mathrm{CD}$ are not only rarely observed. A definite diagnosis of ITB depends on methods that have unsatisfactorily low sensitivities, including $5.3 \%$ to $37.5 \%$ for acid-fast bacilli tissue staining, ${ }^{1-3} 23 \%$ to $46 \%$ for mycobacterial culture, ${ }^{4,5}$ and $36.4 \%$ to $67.9 \%$ for polymerase chain reaction. ${ }^{3,46-8}$ As a result, ITB cannot be confidently excluded-even when all of the above results are negative. In response to this diagnostic dilemma, the current Asia-Pacific guidelines recommend 8-12 weeks of

Received October 30, 2019. Revised February 26, 2020

Accepted March 1, 2020

Correspondence to Julajak Limsrivilai, Division of Gastroenterology,

Department of Medicine, Faculty of Medicine Siriraj Hospital, Mahidol

University, 2 Wanglang Road, Bangkoknoi, Bangkok 10700, Thailand. Tel:

+66-2-419-7281, Fax: +66-2-411-5013, E-mail: alimsrivilai@gmail.com empirical antituberculosis treatment (ATT) for patients with diagnostic uncertainty due to the possible onset of potentially fatal complications if immunosuppressive agents are inappropriately prescribed to ITB patients. ${ }^{9}$ However, 8-12 weeks of empiric ATT can delay appropriate CD treatment, and this can lead to exacerbation of disease and disease-related complications. ${ }^{10}$ Additionally, ATT can cause many side effects, and may facilitate the development of Mycobacterium tuberculosis drug resistance. As a consequence of the difficulty distinguishing between these two conditions, many studies have been conducted that aimed to identify factors that could improve our ability to reliably diagnose these 2 gastrointestinal diseases. Those studies did identify some significant characteristics, but none of those characteristics are exclusive to either ITB or CD. Alternatively, those research teams integrated the factors that they identified with established and more recently developed diagnostic parameters to create diagnostic 
models that could increase the accuracy of diagnosis. However, the number and types of parameters differ among diagnostic models, and the systems used to calculate scoring also vary from model to model. The early models included diagnostic parameters routinely available and used in clinical practice, such as clinical features, endoscopic findings, and pathologic findings. The diagnostic models developed later included more advanced diagnostic parameters, such as high-resolution imaging and serological testing. In this review, we set forth to summarize all of the models designed to differentiate ITB from CD that have been published to date. The search strategy was performed in PubMed database with the search term of ("intestinal" AND "tuberculosis") AND ("Crohn" OR "Crohn's disease"). Only articles reporting a model or a systematic score for differentiating between CD and ITB were included. Fifteen models or scoring systems were identified. The models are classified into the 5 following groups: (1) models that integrate clinical and endoscopy; (2) models that integrate clinical, endoscopy, and pathology; (3) models that integrate clinical, endoscopy, pathology, and imaging; (4) models that integrate clinical, endoscopy, pathology, imaging, and laboratory; and (5) a model that includes adjustable integrated variables. Improved awareness and understanding of the currently available diagnostic models for differentiating CD and ITB may improve diagnosis, treatment, and outcomes.

\section{MODELS INTEGRATING CLINICAL AND ENDOS- COPY}

In 1972, Tandon and Prakash ${ }^{11}$ reported the pathology of ITB and its distinction from CD based on 169 cases (10 CD and 159 ITB) who presented with intestinal obstruction and who underwent intestinal resection. They observed that ITB rarely involved the anus, the length of strictures was generally less than $3 \mathrm{~cm}$, and the ulcers were usually circumferential and generally traveled in transverse axis. In contrast, CD commonly involved the anus, had long segmental stricture, and the ulcers were more prominent along mesenteric attachment and traveled in longitudinal axis. After endoscopy was introduced into routine clinical practice, similar characteristics were reported in endoscopic findings. ${ }^{12,13}$

In 2006, Lee et al. ${ }^{14}$ proposed the first scoring system based on colonoscopic findings (Table 1), ${ }^{6,14-17}$ This system was developed based on data from $44 \mathrm{CD}$ and 44 ITB patients. Lee's system includes 8 endoscopic findings, four of which favor $\mathrm{CD}$, including longitudinal ulcers, aphthous ulcers, cobble- stone appearance, and anorectal involvement. The other 4 favor ITB, including transverse ulcers, scars or pseudopolyps, a patulous ileocecal valve, and involvement of less than 4 of 6 segments of the colon, including the ileocecum, ascending colon, transverse colon, descending colon, sigmoid colon, and anorectum. Each finding favoring $\mathrm{CD}$ is given a score of +1 , and each finding favoring ITB is given a score of -1 . The final score represents the summation of all findings. A diagnosis of $\mathrm{CD}$ will be made if the final score is a positive value, whereas ITB will be diagnosed if the final score is negative. If the final score is zero, the diagnosis is indeterminate. This scoring system was used to evaluate their 88 patients, and they were able to achieve a correct diagnosis in 77 patients (87.5\%). Regarding the remaining patients, 7 (8\%) were misdiagnosed, and 4 (4.5\%) had a score of zero (indeterminate diagnosis). There was no validation cohort in that study. A 2015 study by Mao et al. $^{18}$ reported the accuracy of this endoscopic model to be $66.7 \%$.

Some clinical manifestations were also observed to be different between CD and ITB. ${ }^{1,4,19,20}$ Two diagnostic models based on the integration of clinical and endoscopic findings were developed, both used logistic regression to identify significant parameters, and both constructed their models using a logistic regression equation. Those two models are described as follows.

In 2011, Li et al. ${ }^{15}$ conducted a retrospective study in $130 \mathrm{CD}$ and 122 ITB patients. Multivariate analysis revealed 6 significant clinical parameters, and 6 significant endoscopic parameters as shown in Table 1. They then constructed 2 logistic regression models-one based on clinical parameters, and the other based on endoscopic parameters. The mathematical equation for the clinical model is $P=1 /\left(1+\mathrm{e}^{-\left[0.708+1.409^{*} \text { hematochezi }\right.}\right.$ a+2.798* surgery history $+2.713^{*}$ perianal disease $-4.728^{*}$ pulmonary tuberculosis-2.066*ascites$2.414^{*} \mathrm{PPD}$ skin test $)$, and the one for the endoscopic model is $P=1$ / $\left(1+\mathrm{e}^{-\left[0.283+1.499^{*} \text { rectal involved }+1.753^{*} \text { longitudinal ulcers }+2.787^{*} \text { cobblestone sign- }\right.}\right.$ $1.432^{*}$ ileocecal valve involved- $2.379^{*}$ transverse ulcers-3.343*rodent-like ulcers]). For the

clinical model, a diagnosis cutoff of 0.327 was obtained with a sensitivity, specificity, and accuracy of $90.3 \%, 76.8 \%$, and $83.8 \%$, respectively. For the endoscopic model, a diagnosis cutoff of 0.534 was obtained with a sensitivity, specificity, and accuracy of $82.9 \%, 82.0 \%$, and $82.5 \%$, respectively. There was no validation cohort in that study.

In 2017, Jung et al. ${ }^{17}$ performed a retrospective study in 158 CD and 98 ITB patients. They divided their patients equally into development and validation sets. For model development, clinical, endoscopic, and pathologic parameters were 


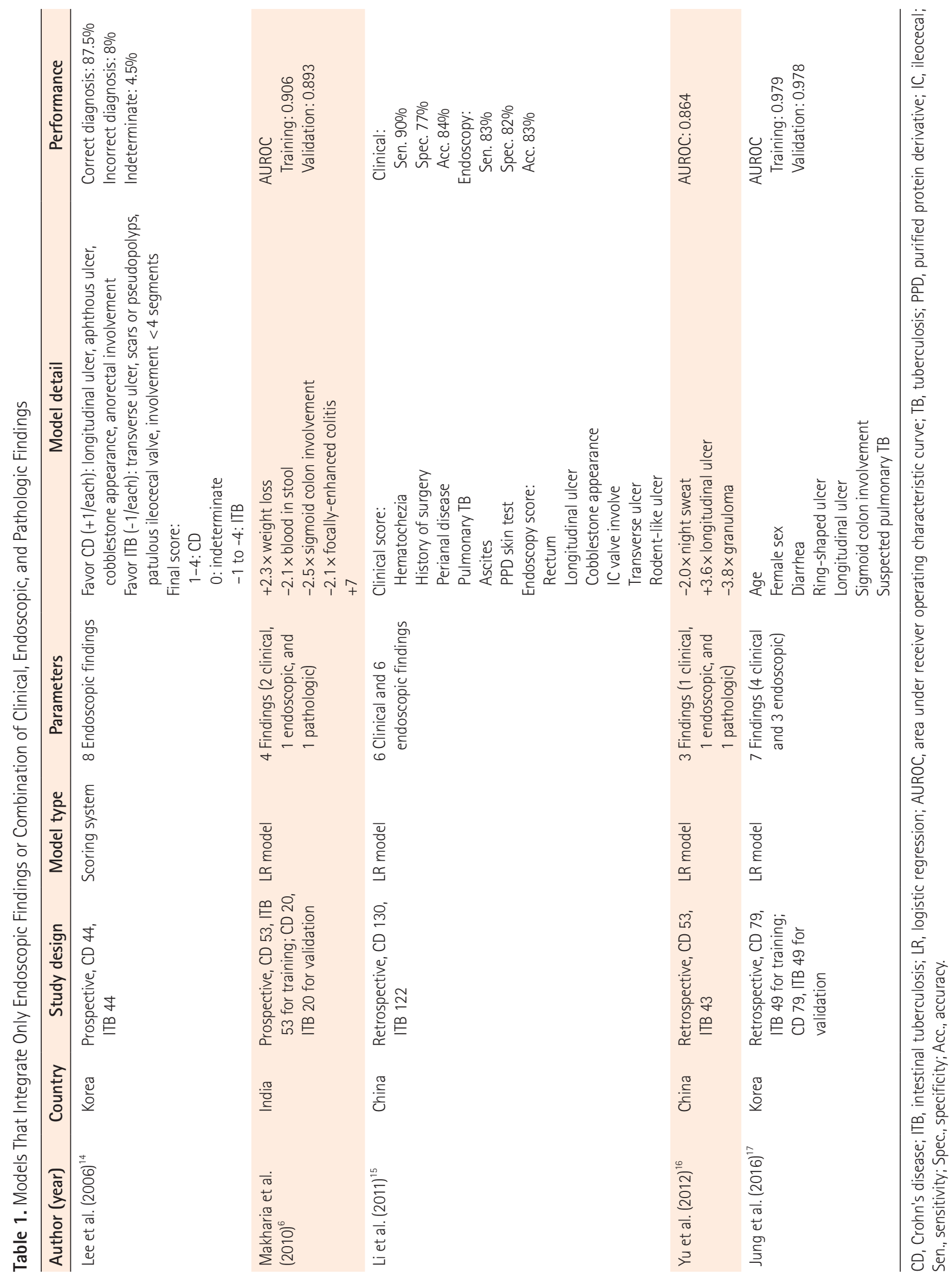


included in univariate analysis, and a receiver operating characteristic (ROC) curve was calculated for each parameter. The factors with an area under the ROC curve (AUROC) of at least 0.7 were selected for inclusion in their model. Five parameters including age, diarrhea, ring-shaped ulcer, longitudinal ulcer, and sigmoid involvement, were identified and included. Two factors with an AUROC curve close to 0.7 (suspicious radiological pulmonary tuberculosis and gender) were also included, because comparative analysis revealed that the 7 -factor model yielded greater diagnostic accuracy than the 5 -factor model. The formula for that model is shown, as follows:

$1 /\left(1+\mathrm{e}^{-\left[-4.423+0.037^{*} \text { age }+2.226^{*} \mathrm{sex}-2.203^{*} \text { diarrhea }+2.345^{*} \text { tran_ring- } 1.911^{*} \text { longitudinal }\right.}\right.$ $-2.123^{*}$ sigmoid $+5.606^{*}$ pul_tbc] $)$.

In the validation set, the sensitivity, specificity, positive predictive value, and negative predictive value at a cutoff level of 0.35 was reported to be $98.0 \%, 92.4 \%, 88.9 \%$, and $98.6 \%$, respectively.

The models integrating clinical and endoscopy are summarized in Table 1.

\section{MODELS INTEGRATING CLINICAL, ENDOSCOPY, AND PATHOLOGY}

Pulimood et al. ${ }^{21}$ reported significant pathologic findings that could differentiate ITB from CD, including microgranuloma and focally-enhanced colitis, which favored a diagnosis of CD, whereas multiple large and confluent granuloma, ulcers lined by conglomerate epithelioid histiocytes, and disproportionate submucosal inflammation favored ITB.

In 2010, Makharia et al. ${ }^{6}$ conducted a prospective study in $53 \mathrm{CD}$ and 53 ITB patients. Clinical manifestations, endoscopic findings, and pathologic findings were included in univariate analysis. Of the parameters in included in subsequent multivariate analysis, 4 significant parameters were identified. Of those, blood in stool, sigmoid colon involvement, and focally-enhanced colitis favored CD, and weight loss favored a diagnosis of ITB. The formula for that model is shown, as follows:

score $=2.3^{*}$ weight loss $-2.1^{*}$ blood in stool $-2.5^{*}$ involvement of sigmoid colon $-2.1^{*}$ focally enhanced colitis +7

A higher score predicted a greater likelihood of ITB. The AUROC value was 0.906 for differentiating CD from ITB in the training cohort. They validated their system in another $20 \mathrm{CD}$ and 20 ITB patients and obtained an AUROC value of 0.893 . At a cutoff value of 5.1, the sensitivity and specificity in the validation model was $90 \%$ and $60 \%$, respectively.
A 2012 retrospective study by Yu et al. ${ }^{16}$ included $53 \mathrm{CD}$ and 43 ITB patients. Multivariable logistic regression analysis revealed night sweats (odds ratio [OR], 0.1; 95\% confidence interval [CI], 0.02-0.1), longitudinal ulcers (OR, 35.5; 95\% CI, 1.8-683.2), and granulomas (OR, 0.02; 95\% CI, 0.002-0.2) to be significant predictors for differentiating $\mathrm{CD}$ from ITB. The model integrating these 3 factors had an AUROC value of 0.8642 (95\% CI, 0.79-0.94), and the formula for that model is shown, as follows:

score $=-2.0$ *night sweat $+3.6 *$ longitudinal ulcers $-3.8^{*}$ granuloma

A higher score predicts a greater likelihood of CD. At a cutoff value of -3.30 , the sensitivity, specificity, and the ability to identify the two diseases correctly was $67.4 \%, 92.5 \%$, and $79.9 \%$, respectively. There was no validation cohort in that study.

The models integrating clinical, endoscopy, and pathology are summarized in Table 1.

\section{MODELS INTEGRATING CLINICAL, ENDOSCOPY, PATHOLOGY, AND CTE}

Cross-sectional imaging is being increasingly used as a diagnostic tool. In 1998, Makanjuola ${ }^{22}$ was the first to report findings that differentiate ITB from CD on conventional CT of the abdomen in 9 CD and 18 ITB. Regarding bowel wall changes, concentric bowel wall thickening of equal to or greater than 6 $\mathrm{mm}$ and mural stratification were found in $\mathrm{CD}$, while concentric bowel wall thickening less than $6 \mathrm{~mm}$ and asymmetrical bowel wall thickening were more often observed in ITB. Mesenteric fibrofatty change and the comb sign were found only in $\mathrm{CD}$, whereas lymphadenopathy larger than $1 \mathrm{~cm}$ and lymph node (LN) with central necrosis were identified only in ITB. Parietal thickening and ascites were found only in ITB.

More recently, a computed tomography enterography (CTE) technique was introduced to better examine bowel wall changes. In 2013, Park and $\mathrm{Lim}^{23}$ reported the findings of CTE in 64 CD and 17 ITB. They found that segmental involvement (6-40 $\mathrm{cm})$, moderate wall thickening (5-9 mm), asymmetrical distribution, fibrofatty change, and comb sign were significantly more often observed in CD. Among those findings, comb sign had the best performance with a sensitivity, specificity, and accuracy in diagnosis of $\mathrm{CD}$ of $74.1 \%, 90.9 \%$, and $76.9 \%$, respectively.

In 2014, Zhao et al. ${ }^{24}$ performed a retrospective study in 141 CD and 47 ITB using logistic regression method to establish 2 models based on the integration of clinical manifestations and 
CTE. The clinical model included hematochezia, perianal disease, positive purified protein derivative (PPD) test, ascites, pulmonary tuberculosis, and night sweats. The mathematical equation for that model is $P=1 /\left(1+\mathrm{e}^{-\left[1.738+1.401^{*} \text { hematochezia }+3.746^{*} \text { per }\right.}\right.$ ianal disease-4.746* positive PPD test-2.022*ascites-1.867* pulmonary TB-3.204*night sweat] ).

The AUROC value for this model was 0.916. At a diagnostic threshold of 0.806, the sensitivity, specificity, and accuracy was $94.3 \%, 80.4 \%$, and $91 \%$, respectively.

The CTE model included left colon involvement, asymmetrical pattern of involvement, abscess, comb sign, LN distribution along the right colic artery, contracture of ileocecal valve, fixed patulous ileocecal valve, and LN with central necrosis. The mathematical equation for that model is $P=1 /(1+\mathrm{e}$ $-\left[-1.525+2.901^{*}\right.$ left colon involvement $+3.925^{*}$ asymmetrical pattern of involvement $+3.441^{*}$ absce ss $+4.539^{*} \mathrm{comb}$ sign $-2.825^{*} \mathrm{LN}$ distribution along right colic artery- $5.367^{*}$ contracture of ileocecal valve-4.264*fixed patulous ileocecal valve-5.059* LN with central necrosis] $)$. The AUROC value for this model was 0.986 . At a diagnostic threshold of 0.682 , the sensitivity, specificity, and accuracy was $96.5 \%$, $93.6 \%$, and $95.7 \%$, respectively. There was no validation cohort in that study.

In 2015, Mao et al. ${ }^{18}$ conducted a prospective study in 105 consecutive patients (67 CD and 38 ITB) who underwent CTE and colonoscopy. Multivariate analysis showed segmental small bowel involvement (OR, 0.104; 95\% CI, 0.022-0.50) and comb sign (OR, 0.02; 95\% CI, 0.003-0.26) to be independent predictors of CD. No significant findings favoring ITB were identified in multivariate analysis. They then added segmental small bowel involvement and comb sign to the endoscopic model by Lee et al. ${ }^{14}$ When the endoscopic score of the Lee et al. model is a positive value, a diagnosis of $\mathrm{CD}$ will be made regardless of CTE findings. However, when the endoscopic score is zero or less, the CTE findings will be included and evaluated. If there is presence of either segmental small bowel involvement or the comb sign, the diagnosis will be changed to CD. Using this algorithm, the accuracy of diagnosis significantly increased from $66.7 \%(70 / 105)$ to $95.2 \%$ $(100 / 105)$ in the development cohort. They then validated their algorithm in 60 new patients (40 CD and 20 ITB), which showed that the additional CTE findings improved the accuracy of diagnosis from $71.6 \%$ when based on the endoscopic score alone to $88.3 \%$ when incorporating CTE findings $(P<0.001)$. Although these results look promising, the limitation of this algorithm is its low specificity. The reported specificity in the validation cohort was only $80 \%$, which means that a significant number of patients with ITB would have been misdiagnosed as CD, and that they would have inappropriate- ly been prescribed immunosuppressive agents.

In 2015, Zhang et al. ${ }^{25}$ performed a prospective study in 92 CD and 31 ITB patients. The model used logistic regression equation based on 6 parameters which were statistically significant in multivariate analysis. The mathematical equation for the model is $P=1 /\left(1+\mathrm{e}^{-\left[-1.279+4.814^{*} \text { perianal disease-5.151*transverse co- }\right.}\right.$ lon $-3.662^{*}$ rodent-like ulcer $+5.399^{*}$ skip lesion $-3.897^{*}$ fixed patulous IC valve $+4.477^{*} \mathrm{comb}$ sign] ).

The AUROC value for this model was 0.994. At a diagnostic threshold of 0.508, the sensitivity, specificity, and accuracy was $97.8 \%, 96.8 \%$, and $97.6 \%$, respectively. There was no validation cohort in this study.

In 2015, Kedia et al. ${ }^{26}$ performed a retrospective study in 44 $\mathrm{CD}$ and 50 ITB patients who underwent CT enteroclysis/ CTE/CT abdomen before starting treatment. Multivariate analysis revealed ileocecal involvement, long segment involvement, and presence of $\mathrm{LN} \geq 1 \mathrm{~cm}$ to be statistically significant factors. The score created based on these findings was calculated using the following formula:

Risk score $=$ long-segment involvement $+(1-$ ileocecal region involvement $)+(1-$ lymph nodes $\geq 1 \mathrm{~cm})$

Scoring for this system ranges from 0 to 3 , and a higher score predicted a greater likelihood of $\mathrm{CD}$. When the score was extremely high or low, its specificity was good. More specifically, if the score was 3 , the specificity for diagnosis of CD was $90 \%$, and if the score was 0 , the specificity for diagnosis of ITB was $100 \%$. However, the sensitivity was poor, as evidenced by the $37 \%$ sensitivity for diagnosis of $\mathrm{CD}$ when the score was 3 , and the $14 \%$ sensitivity for diagnosis of ITB when the score was 0 . Furthermore, the performance of score values of 1 or 2 was found to be unsatisfactory. The sensitivity and specificity for diagnosis of CD when the score was 2 was only $32 \%$ and $50 \%$, respectively. The corresponding values for diagnosis of ITB when the score was 1 was $44 \%$ and $87 \%$, respectively. Although the results of this model demonstrate limitations, the results supported that CTE should play some role in differentiating ITB from CD.

The same research group then conducted another study in CTE findings in 2017. That study found visceral fat (VF) quantification to be a significant surrogate marker for differentiating CD from ITB. ${ }^{27}$ They observed that the VF area and the $\mathrm{VF} /$ subcutaneous fat (SC) ratio were both significantly higher in CD patients than in ITB patients. A cutoff of 0.63 for the $\mathrm{VF} /$ SC ratio had a high sensitivity $(81 \%)$ and specificity $(78 \%)$ for differentiating CD from ITB in the validation cohort. In 2018 that group proposed a new risk score based on CTE findings of $\mathrm{VF} / \mathrm{SC}$ ratio $>0.63$ and long segment involvement $\geq 3 \mathrm{~cm}^{27}$ 
The risk score is defined as follows: $\mathrm{VF} / \mathrm{SC}$ ratio $>0.63+$ long segment involvement $(\geq 3 \mathrm{~cm})$, where $\mathrm{VF} / \mathrm{SC}$ ratio $>0.63=1$ and presence of long segment involvement is $=1$. In their algorithm, patients with necrotic lymph node was diagnosed as ITB regardless of their risk score results. The risk score then was applied to the remaining patients. Among the remaining patients with a score of 2, a diagnosis of CD was made correctly with $50 \%$ sensitivity and $100 \%$ specificity in the validation cohort. Using this algorithm, 43\% of patients (55/128) could obtain a correct diagnosis based on CT alone.

The models integrating clinical, endoscopy, pathology, and CTE are summarized in Table 2. ${ }^{18,24-26,28}$

\section{MODELS INTEGRATING CLINICAL, ENDOSCOPY, PATHOLOGY, IMAGING, AND LABORATORY}

The anti-Saccharomyces cerevisiae antibody (ASCA) has been recognized as a specific serologic marker of CD. ASCA was reported to be positive in about $50 \%$ of $\mathrm{CD}$ patients. ${ }^{29}$ However, the results of studies using ASCA for differentiating ITB from $\mathrm{CD}$ are conflicting. ${ }^{30-32}$ More recently, interferon-gamma release assay (IGRA), which has been available for several years for the diagnosis of latent tuberculosis, has been increasingly used for differentiating ITB from CD. IGRA detects a cell-mediated immune response by measuring in vitro interferon- $\gamma$ production in response to stimulation by antigens derived from M. tuberculosis. Many studies have reported its utility in differentiating ITB from CD in Asians, including a meta-analysis that was conducted in 2014. ${ }^{33}$

In 2015, Huang et al. ${ }^{34}$ performed a prospective study in 25 $\mathrm{CD}$ and 40 ITB patients. They found 16 parameters that were significantly different between CD and ITB in univariate analysis. Of those, 12 parameters with a high specificity ( 7 favored CD, 5 favored ITB) were selected for the development of a scoring system. Each parameter that favors $\mathrm{CD}$ is given a score of +1 , including longitudinal ulcers, nodular hyperplasia, cobblestone appearance, intestinal diseases, intestinal fistulas, target sign, and comb sign. Each parameter that favors ITB is given a score of -1 , including night sweats, positive PPD tests, positive T-SPORTTB (a type of ELISpot assay that is used for tuberculosis diagnosis that belongs to the group of IGRA), ring ulcers, and ulcer scars. The final score represents the summation of all findings. The obtained AUROC value was 0.997 , and at a cutoff of -0.5 , the diagnostic sensitivity, specificity, and accuracy was $100 \%, 95 \%$, and $97 \%$, respectively. Although the score performed very well, the sample size was small and there was no validation cohort in that study.

In 2017, Bae et al. ${ }^{35}$ conducted a prospective study that integrated imaging findings and serology into the previous endoscopic scoring system by Lee et al. There were $40 \mathrm{CD}$ and 40 ITB patients in the development cohort. In addition to colonoscopy, all patients underwent the following investigations: ESR, ASCA IgA and IgG, QuantiFERON-TB Gold In-Tube Test (QFTG; QIAGEN, Hilden, Germany), chest X-ray, and small bowel follow through. All evaluated parameters were compared between CD and ITB patients. Multivariate analysis revealed positive ASCA IgA and/or IgG and proximal intestine involvement (small intestine at least $20 \mathrm{~cm}$ proximal to ileocecal valve) to be independent predictors of $\mathrm{CD}$, and positive QFT-G and typical pulmonary tuberculosis findings to be factors independently associated with ITB. They then integrated these significant parameters into their new model, as shown in Table $3 .{ }^{34-38}$ When the cutoff score was set at 0 or above, the accuracy of the score for diagnosis of CD was $96.3 \%$, with a sensitivity of $95 \%$ and a specificity of $97.5 \%$. The performance of their new model reflected improvement over the model that included only endoscopy. They then validated the model in an additional 37 patients (14 CD and 23 ITB). The AUROC value when the model was applied in the validation group was 0.981 .

In 2018, Wu et al. ${ }^{36}$ performed a prospective study in 239 patients (153 CD and 86 ITB). They randomly divided their study patients into the training set (70\%) and the validation set (30\%). Five parameters were significant in multivariable analysis, including perianal disease, pulmonary involvement, longitudinal ulcer, left colon involvement, and the TB-specific Ag (TBAg)/phytohaemagglutinin (PHA) ratio in T-SPOT.TB. Those 5 parameters were then included in their predictive model that was developed based on logistic regression analysis. The mathematical equation for that model is shown, as follows:

$$
\begin{aligned}
& P=1 /\left(1+\mathrm{e}^{-\left[-1.950-2.372^{*} \text { perianal disease }+2.746^{*} \text { pulmonary involvement }-\right.}\right. \\
& 3.284 \text { *longitudinal ulcer-1.738*left colon+7.477*TBAg/PHA ratio] }
\end{aligned}
$$

When that model was applied to the validation cohort, the AUROC value was 0.95 . At a cutoff value of 0.29 , the sensitivity, specificity, and accuracy was $88.5 \%, 93.5 \%$, and $91.7 \%$, respectively.

A 2019 prospective study by He et al. ${ }^{37}$ included 310 consecutive patients (219 CD and 91 ITB). Of those, 212 patients (143 CD and 69 ITB) were included in the derivation cohort. A prediction model was then developed using a 2-step approach. In the first step, potentially informative variables were identified and ranked based on random forest analysis. The 


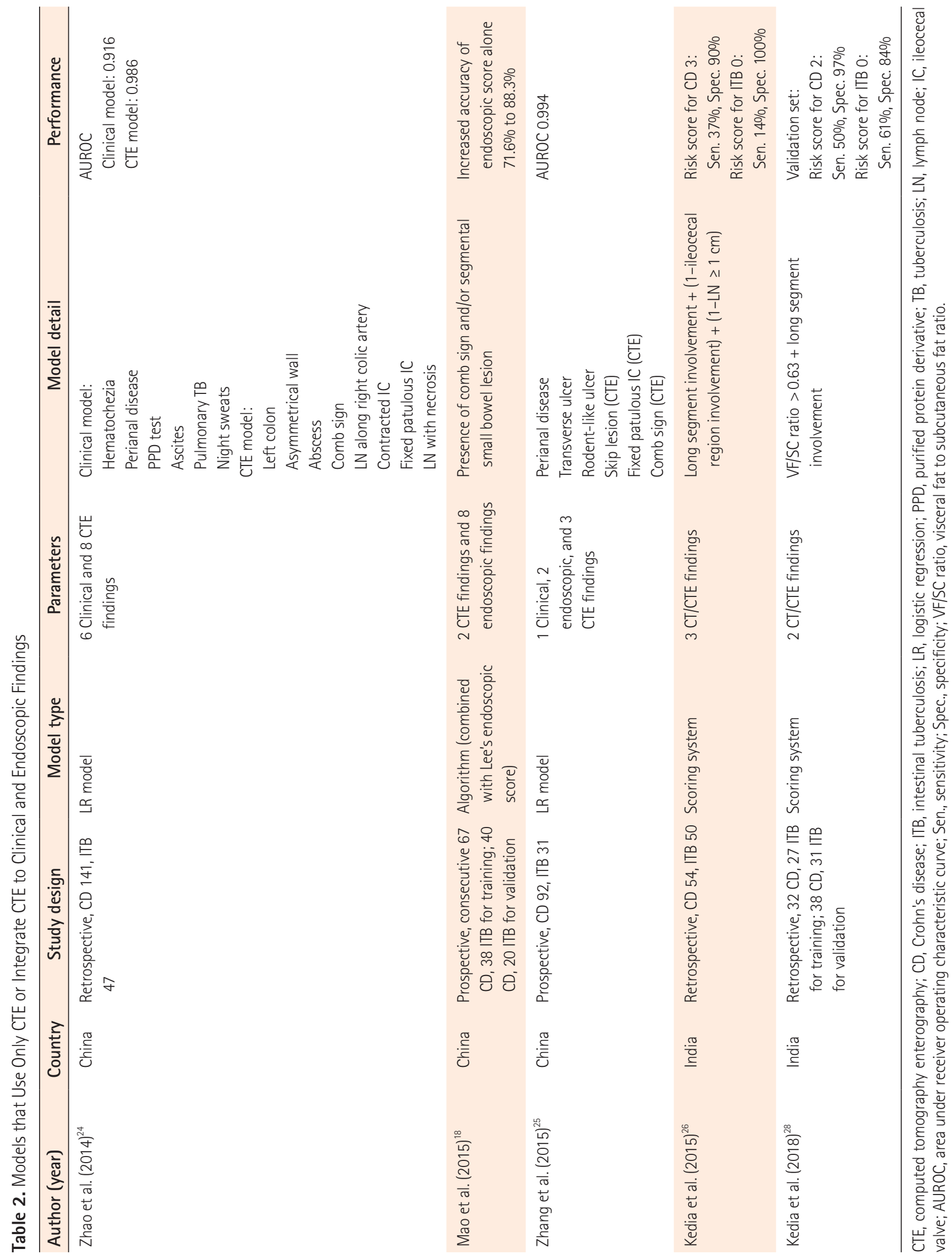




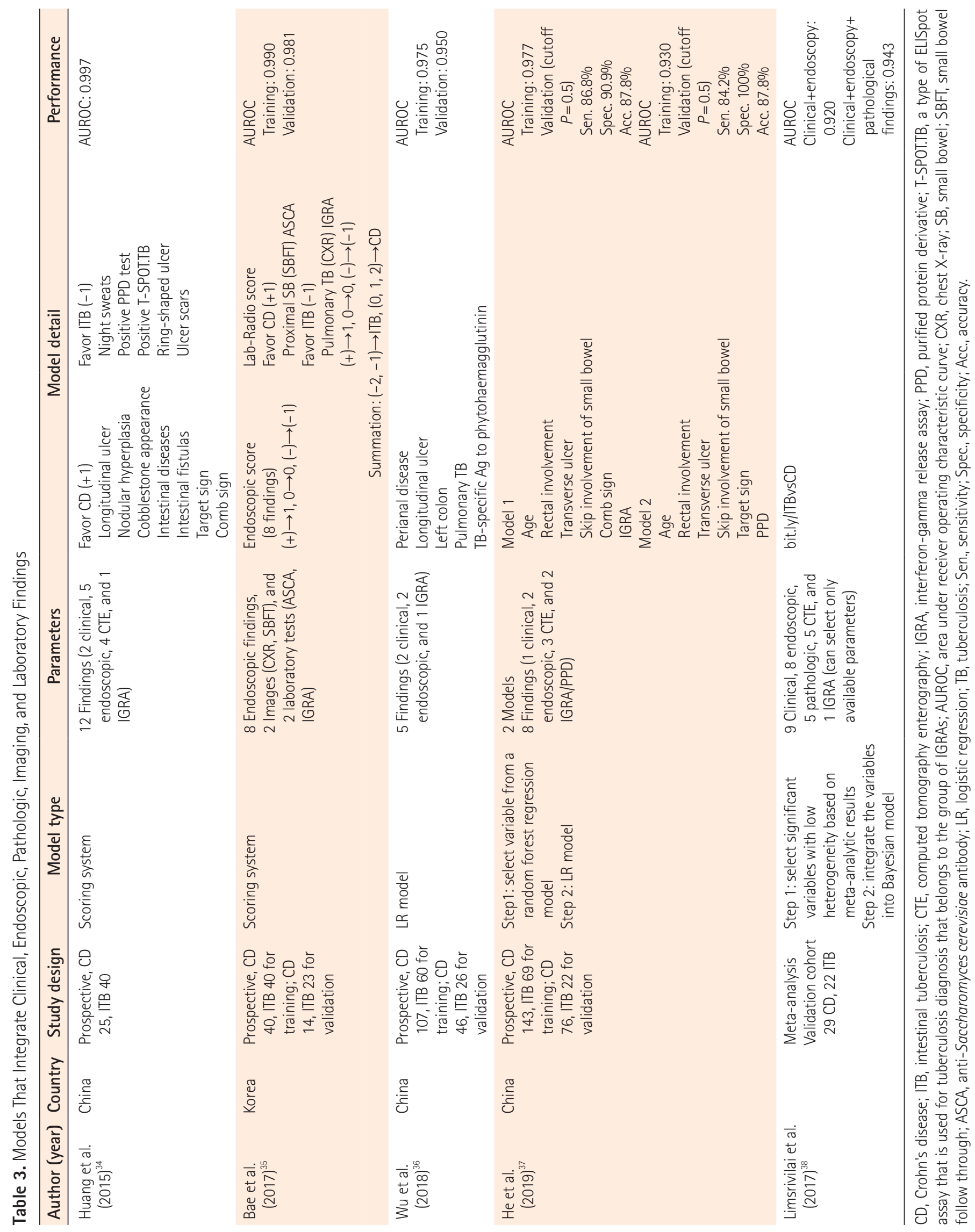


most informative variables were then selected to build the model. Eight variables, including age, rectal involvement, transverse ulcer, skip involvement of the small bowel, target sign on CTE, comb sign on CTE, IGRAs, and PPD test, were selected. For the second step, the selected variables were incorporated into the model using a logistic regression equation. Two models were built. Each shared the same 4 parameters, including age, rectal involvement, transverse ulcer, and skip involvement of the small bowel. The other two variables in model 1 were comb sign and IGRA, and the other two variables in model 2 were target sign and PPD. Nomograms calculating CD probability based on the results of logistic regression were constructed for ease of use. Both models were validated in the other 98 consecutive patients (76 CD and 22 ITB). At a cutoff point of $P=0.5$, the sensitivity, specificity, and accuracy of model 1 was $86.8 \%, 90.9 \%$, and $87.8 \%$, respectively; and the corresponding values for model 2 were $84.2 \%, 100 \%$, and $87.8 \%$, respectively.

The models integrating clinical, endoscopy, pathology, imaging, and laboratory tests are summarized in Table 3.

\section{MODEL WITH ADJUSTABLE INTEGRATED VARIABLES}

In 2017, Limsrivilai et al. ${ }^{38}$ developed a model based on the results of a meta-analysis. Studies that were conducted to differentiate CD from ITB from inception to September 2015 were included. Fifty-five variables that were mentioned in at least 3 studies were selected for meta-analysis. Random effects model was used to determine the significance of each variable. Significant dichotomous variables with low-to-moderate heterogeneity defined by an $I^{2}$ value less than $50 \%$ were selected for development of the model. The model calculated the probability of ITB based on the relative prevalence of ITB versus CD (the pretest probability of ITB, $\mathrm{P}_{0}$ ) and the likelihood ratio (LR) for ITB of each predictor variable in the model. The formula that was developed is shown, as follows: $\mathrm{P}^{\prime}=\left(\mathrm{P}_{0}{ }^{*} \mathrm{LR}\right) /([1-$ $\left.\left.\mathrm{P}_{0}\right]+\left[\mathrm{P}_{0}{ }^{*} \mathrm{LR}\right]\right)$. Using this model, ITB probability can be calculated using only available variables. For any parameters with no available results, the model defaults to an LR of 1 for that parameter, which results in that parameter having no effect on the calculated ITB probability. For example, users can calculate ITB probability with only clinical variables, only endoscopic variables, or a combination of clinical and endoscopic variables. The model was validated in 49 patients (27 CD and 22 ITB). The AUROC value for this model, including clinical and endoscopic variables, was 0.920 , and the AUROC value increased to 0.943 when pathologic data was added. However, this model has some limitations. First, it does not include continuous variables. So, potential variables like age and duration of symptoms cannot be included. Second, the relative prevalence of ITB and CD needs to be input into the model. Third, some parts of the model, such as CTE variables, were based on the results of a small number of studies. Fourth and last, since the model included only studies published before September 2015 , new variables, such as VF/SC ratio, were not included.

A summary of this model is shown in Table 3.

Which model should be used? Model selection should be made after considering the following factors: (1) model performance and validation; (2) availability of data to satisfy the parameters included in the model; or (3) ease of use and thorough understanding of the model.

Surprisingly, no studies have yet been conducted to externally validate any of the models, and there have been no studies that have compared the different models in the same study cohort. All models were reported to have an AUROC value greater than 0.85 . However, only the models by Makharia et al. ${ }^{6}$ Jung et al., ${ }^{17}$ Mao et al.. ${ }^{18}$ Kedia et al., ${ }^{28}$ Bae et al., ${ }^{35}$ Wu et al., ${ }^{36} \mathrm{He}$ et al. ${ }^{37}$ and Limsrivilai et al. ${ }^{38}$ were tested in validation cohorts in their original studies. Among those models, the models by Makharia et al., ${ }^{6}$ Mao et al., ${ }^{18}$ Bae et al., ${ }^{35}$ Wu et al., ${ }^{36}$ and He et al. ${ }^{37}$ were developed and validated based on prospective data.

Model-specific data availability is important. The models that include only clinical and endoscopic findings are the most feasible for use in limited-resource settings, and all gastroenterologists can use these models. This group includes the endoscopic model by Lee et al., ${ }^{14}$ and the models by Li et al., Jung et al., ${ }^{17}$ and Limsrivilai et al. ${ }^{38}$ Even pathologic findings, which are normally routinely obtained, may not be available in all settings due to the lack of GI pathologists in many areas. CTE and serological tests are somewhat beneficial, but one or both are not available in many regions, and these investigations can increase cost of diagnosis. Therefore, in settings where patients have unequal access to diagnostic investigations, the adjustable model developed by Limsrivilai et al. may be most suitable.

Ease of use is an essential characteristic of any model that needs to be used in routine clinical practice. Many research groups developed and proposed models based on logistic regression calculation. ${ }^{6,15-17,24,37}$ Even though the formula can be created and calculated using Microsoft Excel (Microsoft Corp., 
Redmond, WA, USA), they are still potentially and prohibitively complicated, particularly for general physicians who do not perform research and who are unfamiliar with logistic regression. Models based on simple calculations are easier to use than those that use logistic regression analysis. ${ }^{14,27,29}$ The models by Mao et al. ${ }^{18}$ and Bae et al. ${ }^{35}$ give a simple score for each finding and integrate the parameters in a diagnostic algorithm. Although they use complicated formulas and they include many variables, the models by He et al. ${ }^{37}$ and Limsrivilai et al. ${ }^{38}$ were designed for easy use by clinicians. He et al. designed nomograms to calculate the probability of CD, and Limsrivilai et al. designed a website that physicians can use to input data by dropdown menu for each parameter to calculate the probability of ITB. That website can be accessed at bit.ly/ITBvsCD.

\section{CONCLUSION}

Many models have been proposed to differentiate ITB from CD to help decrease the rate of incorrect empirical therapy. The currently available diagnostic models increase the probability of correct diagnosis, appropriate treatment, and improved patient outcomes. Physicians can choose the model that is most appropriate for their use while taking into consideration model performance, availability of parameter data, and ease of use. However-although improvement has been made, none of the currently available models is able to reliably and conclusively able to differentiate CD from ITB. Continued research is, therefore, required. Furthermore and interestingly, none of the models have been validated externally and compared with the other models. External validation and comparison of performance among models in the same cohort is, therefore, warranted.

\section{ADDITIONAL INFORMATION}

\section{Funding Source}

The authors received no financial support for the research, authorship, and/or publication of this article.

\section{Conflict of Interest}

No potential conflict of interest relevant to this article was reported.

\section{Author Contribution}

Conceptualization: Limsrivilai J. Methodology: Limsrivilai J. Writing - original draft: Limsrivilai J. Writing - review and edit- ing: Limsrivilai J, Pausawasdi N. Approval of final manuscript: all authors.

\section{Non-Author Contribution}

The authors gratefully acknowledge Kevin P. Jones (Siriraj Medical Research Center, Faculty of Medicine, Siriraj Hospital, Mahidol University, Bangkok, Thailand) for language editing.

\section{ORCID}

$\begin{array}{ll}\text { Limsrivilai J } & \text { https://orcid.org/0000-0001-5867-0312 } \\ \text { Pausawasdi N } & \text { https://orcid.org/0000-0002-3737-8555 }\end{array}$

\section{REFERENCES}

1. Dutta AK, Sahu MK, Gangadharan SK, Chacko A. Distinguishing Crohn's disease from intestinal tuberculosis: a prospective study. Trop Gastroenterol 2011;32:204-209.

2. Li Y, Zhang LF, Liu XQ, et al. The role of in vitro interferon $\gamma$ release assay in differentiating intestinal tuberculosis from Crohn's disease in China. J Crohns Colitis 2012;6:317-323.

3. Lei Y, Yi FM, Zhao J, et al. Utility of in vitro interferon- $\gamma$ release assay in differential diagnosis between intestinal tuberculosis and Crohn's disease. J Dig Dis 2013;14:68-75.

4. Amarapurkar DN, Patel ND, Rane PS. Diagnosis of Crohn's disease in India where tuberculosis is widely prevalent. World J Gastroenterol 2008;14:741-746.

5. Ramadass B, Chittaranjan S, Subramanian V, Ramakrishna BS. Fecal polymerase chain reaction for Mycobacterium tuberculosis IS6110 to distinguish Crohn's disease from intestinal tuberculosis. Indian J Gastroenterol 2010;29:152-156.

6. Makharia GK, Srivastava S, Das P, et al. Clinical, endoscopic, and histological differentiations between Crohn's disease and intestinal tuberculosis. Am J Gastroenterol 2010;105: 642-651.

7. Jin XJ, Kim JM, Kim HK, et al. Histopathology and TB-PCR kit analysis in differentiating the diagnosis of intestinal tuberculosis and Crohn's disease. World J Gastroenterol 2010;16:24962503.

8. Fei BY, Lv HX, Zheng WH. Fluorescent quantitative PCR of Mycobacterium tuberculosis for differentiating intestinal tuberculosis from Crohn's disease. Braz J Med Biol Res 2014;47: 166-170.

9. Ooi CJ, Makharia GK, Hilmi I, et al. Asia Pacific Consensus Statements on Crohn's disease. Part 1: Definition, diagnosis, and epidemiology. J Gastroenterol Hepatol 2016;31:45-55.

10. Banerjee R, Pal P, Girish BG, Reddy DN. Risk factors for diag- 
nostic delay in Crohn's disease and their impact on longterm complications: how do they differ in a tuberculosis endemic region? Aliment Pharmacol Ther 2018;47:1367-1374.

11. Tandon HD, Prakash A. Pathology of intestinal tuberculosis and its distinction from Crohn's disease. Gut 1972;13:260269.

12. Chutkan RK, Scherl E, Waye JD. Colonoscopy in inflammatory bowel disease. Gastrointest Endosc Clin N Am 2002;12: 463-483.

13. Shah S, Thomas V, Mathan M, et al. Colonoscopic study of 50 patients with colonic tuberculosis. Gut 1992;33:347-351.

14. Lee YJ, Yang SK, Byeon JS, et al. Analysis of colonoscopic findings in the differential diagnosis between intestinal tuberculosis and Crohn's disease. Endoscopy 2006;38:592-597.

15. Li X, Liu X, Zou Y, et al. Predictors of clinical and endoscopic findings in differentiating Crohn's disease from intestinal tuberculosis. Dig Dis Sci 2011;56:188-196.

16. Yu H, Liu Y, Wang Y, Peng L, Li A, Zhang Y. Clinical, endoscopic and histological differentiations between Crohn's disease and intestinal tuberculosis. Digestion 2012;85:202-209.

17. Jung Y, Hwangbo Y, Yoon SM, et al. Predictive factors for differentiating between Crohn's disease and intestinal tuberculosis in Koreans. Am J Gastroenterol 2016;111:1156-1164.

18. Mao R, Liao WD, He Y, et al. Computed tomographic enterography adds value to colonoscopy in differentiating Crohn's disease from intestinal tuberculosis: a potential diagnostic algorithm. Endoscopy 2015;47:322-329.

19. Gu Q, Ouyang Q, Zhang WY, Li GD. A comparison of clinical and pathologic characteristics between Crohn's disease and intestinal tuberculosis. Zhonghua Nei Ke Za Zhi 2009;48:291294.

20. Pulimood AB, Amarapurkar DN, Ghoshal U, et al. Differentiation of Crohn's disease from intestinal tuberculosis in India in 2010. World J Gastroenterol 2011;17:433-443.

21. Pulimood AB, Ramakrishna BS, Kurian G, et al. Endoscopic mucosal biopsies are useful in distinguishing granulomatous colitis due to Crohn's disease from tuberculosis. Gut 1999;45: $537-541$.

22. Makanjuola D. Is it Crohn's disease or intestinal tuberculosis? CT analysis. Eur J Radiol 1998;28:55-61.

23. Park MJ, Lim JS. Computed tomography enterography for evaluation of inflammatory bowel disease. Clin Endosc 2013; 46:327-366.

24. Zhao XS, Wang ZT, Wu ZY, et al. Differentiation of Crohn's disease from intestinal tuberculosis by clinical and CT enterographic models. Inflamm Bowel Dis 2014;20:916-925.
25. Zhang T, Fan R, Wang Z, et al. Differential diagnosis between Crohn's disease and intestinal tuberculosis using integrated parameters including clinical manifestations, T-SPOT, endoscopy and CT enterography. Int J Clin Exp Med 2015;8:1757817589.

26. Kedia S, Sharma R, Nagi B, et al. Computerized tomographybased predictive model for differentiation of Crohn's disease from intestinal tuberculosis. Indian J Gastroenterol 2015;34: 135-143.

27. Yadav DP, Madhusudhan KS, Kedia S, et al. Development and validation of visceral fat quantification as a surrogate marker for differentiation of Crohn's disease and intestinal tuberculosis. J Gastroenterol Hepatol 2017;32:420-426.

28. Kedia S, Madhusudhan KS, Sharma R, et al. Combination of increased visceral fat and long segment involvement: development and validation of an updated imaging marker for differentiating Crohn's disease from intestinal tuberculosis. J Gastroenterol Hepatol 2018;33:1234-1241.

29. Herszényi L, Tulassay Z. The role of autoantibodies in inflammatory bowel disease. Dig Dis 2012;30:201-207.

30. Ghoshal UC, Ghoshal U, Singh H, Tiwari S. Anti-Saccharomyces cerevisiae antibody is not useful to differentiate between Crohn's disease and intestinal tuberculosis in India. J Postgrad Med 2007;53:166-170.

31. Makharia GK, Sachdev V, Gupta R, Lal S, Pandey RM. AntiSaccharomyces cerevisiae antibody does not differentiate between Crohn's disease and intestinal tuberculosis. Dig Dis Sci 2007;52:33-39.

32. Kim YS, Kim YH, Kim WH, et al. Diagnostic utility of anti-Saccharomyces cerevisiae antibody (ASCA) and interferon- $\gamma$ assay in the differential diagnosis of Crohn's disease and intestinal tuberculosis. Clin Chim Acta 2011;412:1527-1532.

33. Ng SC, Hirai HW, Tsoi KK, et al. Systematic review with metaanalysis: accuracy of interferon-gamma releasing assay and anti-Saccharomyces cerevisiae antibody in differentiating intestinal tuberculosis from Crohn's disease in Asians. J Gastroenterol Hepatol 2014;29:1664-1670.

34. Huang X, Liao WD, Yu C, et al. Differences in clinical features of Crohn's disease and intestinal tuberculosis. World J Gastroenterol 2015;21:3650-3656.

35. Bae JH, Park SH, Ye BD, et al. Development and validation of a novel prediction model for differential diagnosis between Crohn's disease and intestinal tuberculosis. Inflamm Bowel Dis 2017;23:1614-1623.

36. Wu X, Huang H, Hou H, et al. Diagnostic performance of a 5-marker predictive model for differential diagnosis between 
intestinal tuberculosis and Crohn's disease. Inflamm Bowel Dis 2018;24:2452-2460.

37. He Y, Zhu Z, Chen Y, et al. Development and validation of a novel diagnostic nomogram to differentiate between intestinal tuberculosis and Crohn's disease: a 6-year prospective multicenter study. Am J Gastroenterol 2019;114:490-499.

38. Limsrivilai J, Shreiner AB, Pongpaibul A, et al. Meta-analytic Bayesian model for differentiating intestinal tuberculosis from Crohn's disease. Am J Gastroenterol 2017;112:415-427. 